\title{
Generating scattering dark states through the Fano interference between excitons and an individual silicon nanogroove
}

\author{
Jiahao Yan, Churong Ma, Pu Liu, Chengxin Wang and Guowei Yang
}

Effective interactions between excitons and resonating nanocavities are important for many emerging applications in nanophotonics. Although plasmonic nanocavities are considered promising substitutes for diffraction-limited dielectric microcavities, their practical applications are hindered by large ohmic loss and Joule heating. Other than plasmonic materials, highrefractive-index dielectric nanocavities is a new way to trap light in subwavelength scales. However, studies on the interaction between dielectric nanocavities and excitons are still scarce. Here, for the first time, we demonstrate that the Fano interference between molecular excitons and an individual silicon nanogroove can generate scattering dark modes. By placing $\mathrm{J}$-aggregate excitons into a silicon nanogroove, the leaky magnetic resonant modes filling in the groove can tailor their scattering directivity and reduce the uncoupled radiation decay in a specific direction. This unidirectional 'dark state' brings a new approach to tailor the interaction between excitons and nanocavities without large near-field enhancement. By adjusting the resonant modes, the scattering spectra can change from a Fano asymmetric line shape to a significantly suppressed scattering dip. These findings indicate that silicon nanogrooves can provide a platform for integrated on-chip silicon-exciton hybrid optical systems in the future.

Light: Science \& Applications (2017) 6, e16197; doi:10.1038/lsa.2016.197; published online 27 January 2017

Keywords: excitons; light-matter interactions; magnetic mode; silicon nanogroove

\section{INTRODUCTION}

Strong interactions between nanocavities and excitons is important for both fundamental studies and designing functional nanophotonics devices $^{1}$. Bringing together plasmonic nanocavities and molecular excitons results in strong light-matter interactions because of the high density of electromagnetic modes and light trapping in deep subwavelength scales ${ }^{2,3}$. Recently, many groups have studied plasmonexciton coupling systems based on gold or silver ${ }^{4-7}$. However, the advantages of plasmonic nanocavities also hinder the realization of simultaneous effective coupling. On the one hand, the strong nearfield enhancement leads to the high photothermal conversion and Joule heating of the local environment ${ }^{8}$. Temperatures higher than $60^{\circ} \mathrm{C}$ lead to J-aggregates further growing to larger crystallites ${ }^{9}$, which influences the stability of the hybrid system. On the other hand, although plasmonic nanocavities are able to confine light to small mode volumes called 'hot spots', these confined 'hot spots' prevent more effective overlap between the surrounding J-aggregates and localized plasmon modes ${ }^{10}$.

As an alternative, high-index dielectric nanocavities that can manipulate light at a subwavelength scale owing to Mie magnetic and electric resonances ${ }^{11-15}$ have shown promising applications, such as surface-enhanced spectroscopies ${ }^{8}$ and third-harmonic generation ${ }^{16}$. The magnetic resonances in silicon structures are driven by the electric field of light that couples to the displacement of current loops. The interaction between magnetic and electric modes in silicon nanostructures can generate directional scattering ${ }^{17-19}$, which brings new insights into zero-index metamaterials ${ }^{20,21}$, Fano resonances ${ }^{22,23}$ and biosensing ${ }^{24}$. If the magnetic responses can interact with the surrounding excitons, then interesting scattering behaviors may occur. Unfortunately, these modes are internally distributed in silicon nanodisks or nanospheres, which prevent effective interactions with excitons.

Here, for the first time, we demonstrate tunable Fano interferences between excitons (J-aggregates) and individual silicon nanogrooves (NGs). Silicon NGs as slot waveguides have been utilized to overcome the diffraction limit of optical manipulation ${ }^{25}$. They are different from traditional microcavities in that they confine light on at least a halfwavelength scale owing to the presence of mirrors or surrounding photonic crystals ${ }^{1}$. We also establish that silicon NGs hold different resonant modes compared with silicon nanodisks or nanoblocks ${ }^{26}$, that is, leaky magnetic resonant mode (LMRM). Unlike the internal modes in the usual Mie resonators, the LMRM is able to leak out and

State Key Laboratory of Optoelectronic Materials and Technologies, Nanotechnology Research Center, School of Materials Science and Engineering, Sun Yat-sen University, Guangzhou, Guangdong 510275, China

Correspondence: G Yang, Email: stsygw@mail.sysu.edu.cn

Received 19 April 2016; revised 10 August 2016; accepted 10 August 2016; accepted article preview online 12 August 2016 
fill in the groove, which is beneficial for realizing an effective lightmatter interaction. The LMRMs 'shield' J-aggregates from the incident light backward and direct the scattering light mainly forward. In this way, a unidirectional scattering dark state ${ }^{27}$ can be observed, which significantly suppresses backward scattering at the specific wavelength. Using Kerker's scattering theory and a coupled-oscillator model ${ }^{28-30}$, we theoretically explain why silicon NGs can realize prominent Fano interference with excitons without large near-field enhancement and why this scattering dark state can be generated only in silicon NGs and not in other silicon-based nanocavities. Silicon NGs guiding the radiation via strongly confined magnetic resonant modes provide a path for designing various types of integrated nanophotonics devices based on their interactions with excitons ${ }^{31-33}$.

\section{MATERIALS AND METHODS}

\section{Fabrication of silicon NGs}

Silicon NGs with different geometries were fabricated by focused ion beam FIB (FIB-SEM crossbeam workstation, Zeiss Auriga-4525) milling techniques on a single-crystal silicon substrate. The beam current was set to $10 \mathrm{pA}$ for high-resolution structuring. The width and depth of the silicon NGs were determined by top and $85^{\circ}$-tilted SEM (scanning electron microscope), respectively. Before spin-coating, the silicon substrate was treated by ultrasonic cleaning and plasma cleaning to improve its hydrophilicity. TDBC (5,6-dichloro-2-[[5,6dichloro-1-ethyl-3-(4-sulfobutyl)-benzimidazol-2-ylidene]-propenyl]1-ethyl-3-(4-sulfobutyl)-benzimidazolium hydroxide, inner salt, sodium salt) J-aggregates were formed by adding $0.25 \mathrm{~mol} / \mathrm{l} \mathrm{Na} \mathrm{NO}_{4}$ to $0.1 \mathrm{mM}$ TDBC monomers (FEW Chemicals $\mathrm{GmbH}$, Wolfen, Germany) after 10 min ultrasonic treatment. The J-aggregates were then spin-coated on patterned silicon substrate (3000 r.p.m. for $60 \mathrm{~s}$ ), forming a J-aggregate thin film. The thickness was confirmed using atomic force microscopy (AFM). For comparison, silicon nanospheres with diameters from 100 to $200 \mathrm{~nm}$ were fabricated using the method reported in our former work ${ }^{17,22}$.

\section{Dark-field measurements}

The dark-field backward scattering spectra were obtained using a darkfield optical microscope (BX51, Olympus) integrated with a spectrograph (IsoPlane 160, Princeton Instruments) and a charge-coupled device (CCD) camera (PiXis 400B-eXcelon, Princeton Instruments). During the measurements, the oblique incident white light was illuminated with a $53^{\circ}$ incident angle on the entire nanostructures. The scattered light from a certain section of the silicon NG was collected through a dark-field objective on top (numerical aperture $=0.80$ ) and a slit in front of the spectrograph.

\section{Numerical calculations}

The finite difference time domain method was used to calculate the scattering spectra, near-field distributions and far-field scattering patterns of the silicon NGs and hybrid systems. Four total-field scattered-field area sources with $53^{\circ}$ incident angles from four sides at visible wavelengths (300-900 nm) were used to simulate the dark-field scattering in an experiment. Geometrical parameters were obtained from SEM images of each of the NGs. The interaction between the J-aggregates and the NG was simulated by setting 10 -nm-thick J-aggregates in the top side of the grooves. The dielectric function of the silicon was obtained from Palik. The dielectric permittivity of the J-aggregates was described by the Lorentz model as

$$
\varepsilon_{\mathrm{J}}(\omega)=\varepsilon_{\infty}+\frac{f \omega_{0}^{2}}{\left(\omega_{0}^{2}-\omega^{2}-i \gamma_{0} \omega\right)}
$$

where $\varepsilon_{\infty}=1$ is the high-frequency component, $f=0.1$ is the reduced oscillator strength, $\omega_{0}=3.21 \times 10^{15} \mathrm{rad} / \mathrm{s}$ is the exciton transition frequency and $\gamma_{0}=3.0 \times 10^{13} \mathrm{rad} / \mathrm{s}$ is the exciton linewidth.

\section{RESULTS AND DISCUSSION}

In our case, silicon NGs with different sizes were fabricated by FIB milling on a single-crystal silicon substrate (Supplementary Fig. S1). A typical silicon NG with a width of $\sim 100 \mathrm{~nm}$ is shown in Figure 1a. The longitudinal section graph in Figure $1 \mathrm{~b}$ indicates that the NG possesses a tapering shape and that the rate of narrowing becomes faster at the bottom. The dark-field scattering image in Figure 1c embodies a bright scattering light, with a broad resonant spectrum in the visible region. As for a silicon nanosphere, the strongest resonant mode is the magnetic dipole resonance generated from the displacement current loops (see Supplementary Fig. S2). This nanostructure can be explained by Mie theory. Although the scattering from other silicon nanostructures such as cubic and conical shapes cannot be described by Mie theory, they still possess the displacement current loops and the induced magnetic responses ${ }^{34,35}$. To confirm whether silicon NGs also possess the magnetic response, we simulated the scattering spectrum from a silicon NG and the magnetic field distributions on the vertical cross-section at different wavelengths shown in Figure 1d. The pink curve in Figure 1d is the measured absorption spectrum of the TDBC J-aggregating dyes. At $\lambda=365 \mathrm{~nm}$, the scattering light is generated mainly from the surface, and no internal modes were excited. Above $\lambda=400 \mathrm{~nm}$, the light field begins to penetrate into the silicon along the groove and generate a magnetic response. Then, at $\lambda=587 \mathrm{~nm}$ (the absorption peak of J-aggregate) and $\lambda=698 \mathrm{~nm}$, the internal magnetic resonances become the dominant mode. The schematic graph in Figure le shows the distribution of the magnetic field (bright dots) inside the groove, and the white plane $(y-z$ plane $)$ represents the investigated vertical cross-section. The scattering spectra under different polarization directions shown in Figure 1f indicate that the scattering intensity excited by vertical polarization is much stronger. Therefore, we can use the magnetic field distribution (Figure $1 \mathrm{~g}$ ) and electric vector distribution (Figure $1 \mathrm{~h}$ ) under vertically polarized excitation to analyze the resonant mode. Owing to the nanoscale gap, the former regular internal electromagnetic distribution in the silicon slab is damaged, and the magnetic field expands into the top of the NG, which we called LMRM. The electric vector distribution shown in Figure $1 \mathrm{~h}$ is similar to that observed in the silicon nanosphere (Supplementary Fig. S2), which represents the capability to generate an obvious magnetic response.

Comparing the electric and magnetic field distributions at $\lambda=587 \mathrm{~nm}$ between a silicon NG and plasmonic NGs (Supplementary Fig. S3), we can find that the near-field enhancements of gold or silver NGs mainly distribute on the surface. For a silicon NG, however, the resonant modes can be excited inside; therefore, the electromagnetic fields distributed in the groove mainly leak from the bilateral internal resonant modes. The field distributions in Supplementary Fig. S3 also indicate that the field enhancements are much stronger in plasmonic NGs than they are in the silicon NGs. The absorption performance can be evaluated by using a simple formula $P_{\mathrm{abs}}=(1 / 2) \omega \varepsilon^{\prime \prime}|E|^{2}$, where $\omega$ is the frequency, $\varepsilon^{\prime \prime}$ is the imaginary part of permittivity and $|E|$ is the total electric field ${ }^{36,37}$. Through the curves of the imaginary part of permittivity (Supplementary Fig. S4) ${ }^{38}$ and the electric field enhancement amplitude obtained from Supplementary Fig. S3, we can calculate the absorptivity of different types of NGs, and the average absorptivity of the silicon NG is $2 \%$ of the gold NG and $2.3 \%$ of the silver NG. This result confirms the low-loss feature of the silicon 


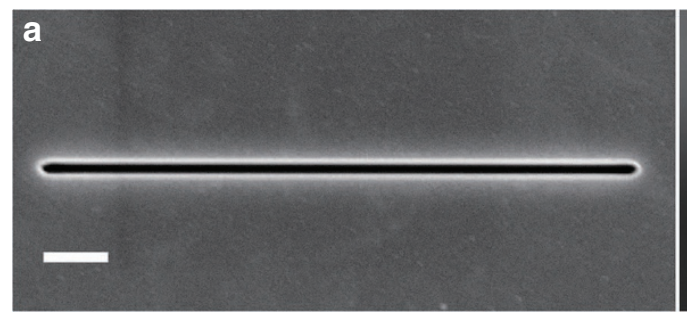

d
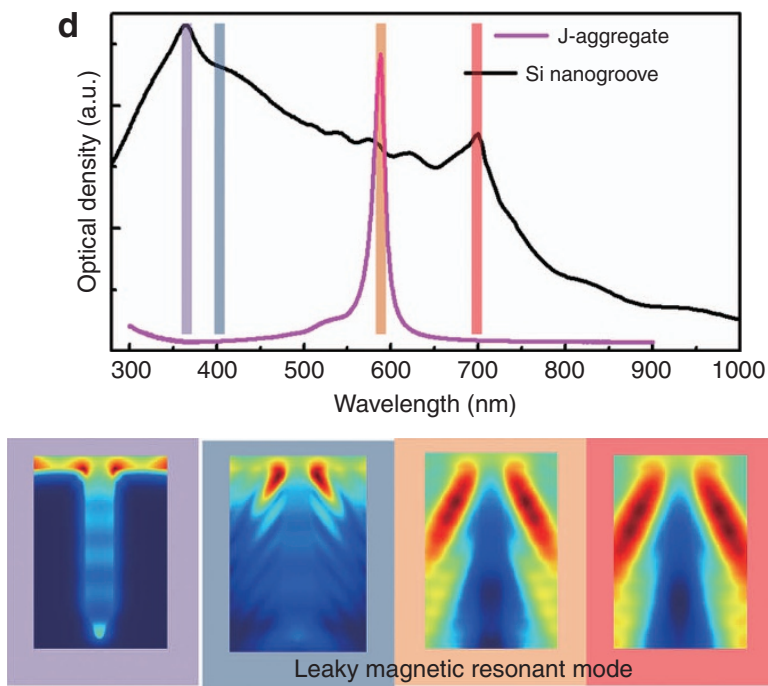

Leaky magnetic resonant mode

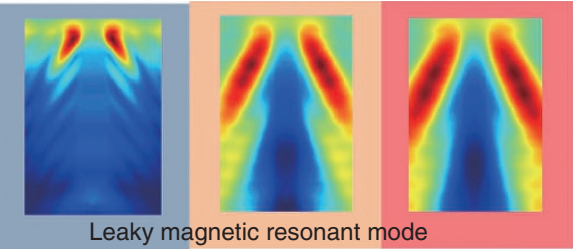

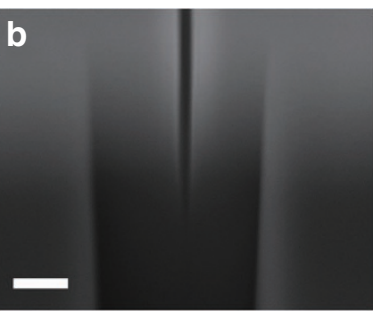

e
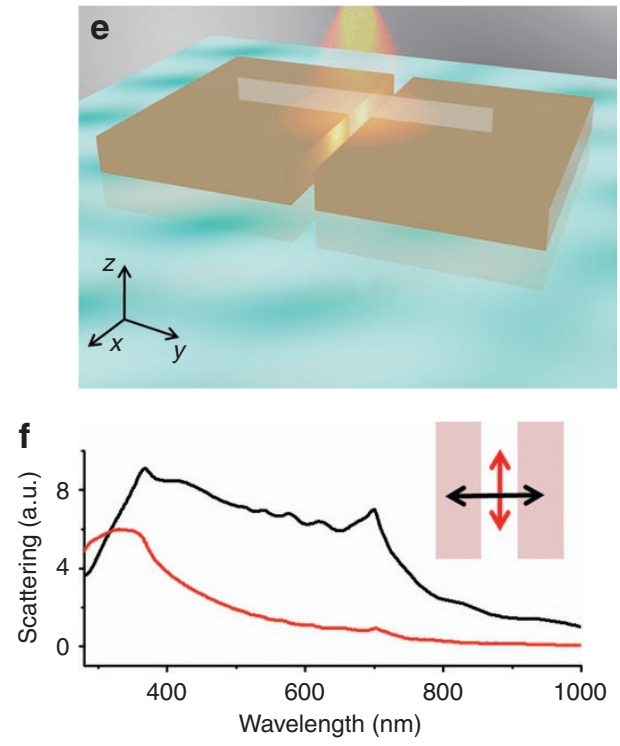
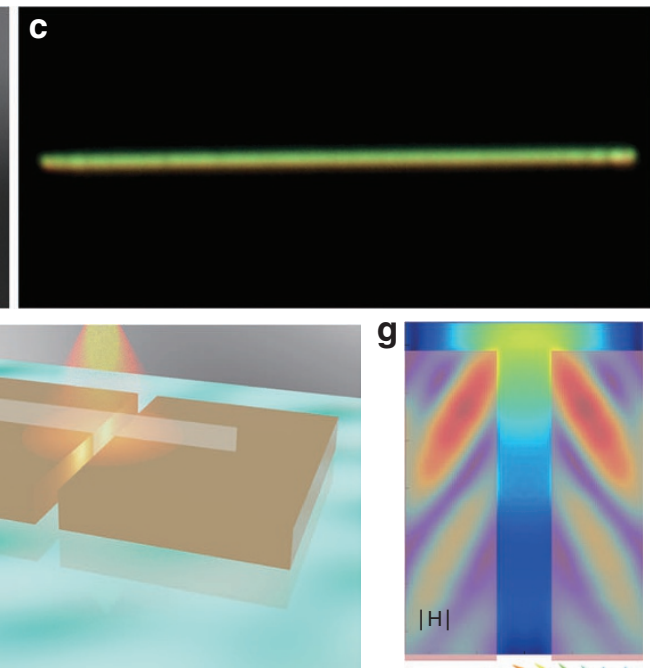

h

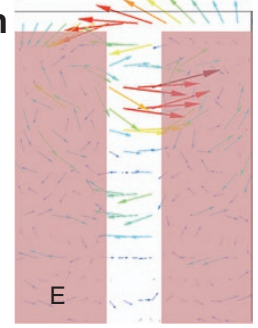

Figure 1 Resonant modes in an individual silicon NG. (a) Top-view SEM image of a fabricated silicon NG with a width of 100 nm and a length of $5 \mu$ m. Scale bar $=500 \mathrm{~nm}$. (b) The tilted SEM image of a typical silicon NG with a depth of $800 \mathrm{~nm}$ (scale bar=300 nm). (c) Dark-field scattering image of a typical silicon NG with a length of $20 \mu \mathrm{m}$. (d) The calculated scattering spectrum of a typical silicon NG and the absorption spectrum of TDBC J-aggregates. Longitudinal section magnetic field distributions in boxes reveal the resonant modes at specific wavelengths. The field distributions and the corresponding locations in the spectrum are marked in the same color. (e) A schematic shows the generation of LMRMs inside the groove. The white plane reveals the investigated $y-z$ plane. (f) Scattering spectra of the silicon NG under different polarization directions. The black curve represents the spectrum under $y$-axis polarization, and the red curve represents the spectrum under $x$-axis polarization. (g) Magnetic field distribution under $y$-axis polarization. (h) Electric field lines under $y$-axis polarization.

nanostructures. Admittedly, the densities of the electromagnetic modes in the silicon NGs are much lower than are those in the plasmonic NGs; therefore, there should be a new mechanism in the interacting process with excitons.

To generate a scattering dark state in a silicon NG-J-aggregate hybrid system, the dimensions of the silicon NG should first be carefully designed to adjust the resonant peak overlapping with the exciton energy. By adjusting the depth and width of the silicon NGs, the scattering behaviors are changed. For a shallow groove with a depth of $100 \mathrm{~nm}$, the resonant peak at approximately $\lambda=500 \mathrm{~nm}$ generated from the surface scattering is dominated as shown in Figure 2a (black curve). At the same time, the magnetic field distribution at $\lambda=587 \mathrm{~nm}$ in Figure $2 \mathrm{~b}$ indicates that the magnetic resonance is formed. When increasing the depth to $150 \mathrm{~nm}$, the usual surface scattering is weakened, but the scattering at longer wavelengths generated from the LMRMs is enhanced (see red curve in Figure 2a). This enhanced scattering occurs because of the larger mode volume of the LMRMs. As shown in Figure 2b, the LMRM can fully fill in the groove. Further increasing the depth brings different distributions of the LMRM and then engineers the resonant peaks. From $d=150$ to $d=300 \mathrm{~nm}$, the mode volumes of the LMRMs at $\lambda=587 \mathrm{~nm}$ first decrease and then slightly increase, which is in accordance with the scattering fluctuation at $\lambda=587 \mathrm{~nm}$ in Figure 2a. For a silicon NG with a depth of more than $400 \mathrm{~nm}$, the distributions of the LMRMs become stable. In addition, the scattering spectra remain unchanged when increasing the depth further (see Figure 2a). The scattering spectra of the silicon NGs calculated using the finite difference time domain method (Figure 2c) agree well with the experimental darkfield observation. The detailed comparison between the magnetic field distributions of the silicon NGs with different depths $(d=150$ and $200 \mathrm{~nm}$ ) in Supplementary Fig. S5 illustrates how LMRMs influence the scattering intensities. For a NG with a depth of $150 \mathrm{~nm}$, the groove always fills the magnetic fields at $\lambda=587$ and $\lambda=700 \mathrm{~nm}$ (point $\mathrm{A}$ in Figure 2c); therefore, the scattering intensities at these two wavelengths are almost the same. For a NG with a depth of $200 \mathrm{~nm}$, the leaky magnetic field is more obvious at $700 \mathrm{~nm}$ (point B in Figure 2c) than at $587 \mathrm{~nm}$; therefore, the scattering intensity is weaker at $587 \mathrm{~nm}$ than it is at $700 \mathrm{~nm}$. Because of our system limitations, we show our experimental data in the range of 400-1000 $\mathrm{nm}$. The lower quantum efficiency of the CCD below $\lambda=500 \mathrm{~nm}$ and the visible-range incident light lead to the quick drop of the light-source spectrum approaching $\lambda=400 \mathrm{~nm}$ (see Supplementary Fig. S6). To simulate this phenomenon well, the raw simulated spectra below $500 \mathrm{~nm}$ have multiplied coefficients to simulate the scattering behavior below $500 \mathrm{~nm}$ more precisely (see Figure 2c). Other deviations between the simulation and experiment may arise from different incident situations. A 'dark-field circle' (Supplementary Fig. S6a) was used to realize the oblique incidence in the experiment, and four oblique incident plane waves 
a

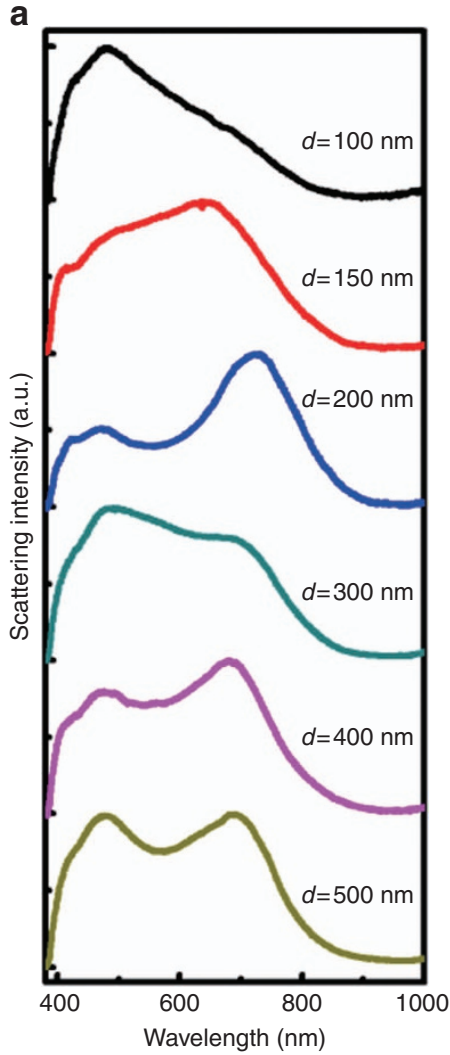

b

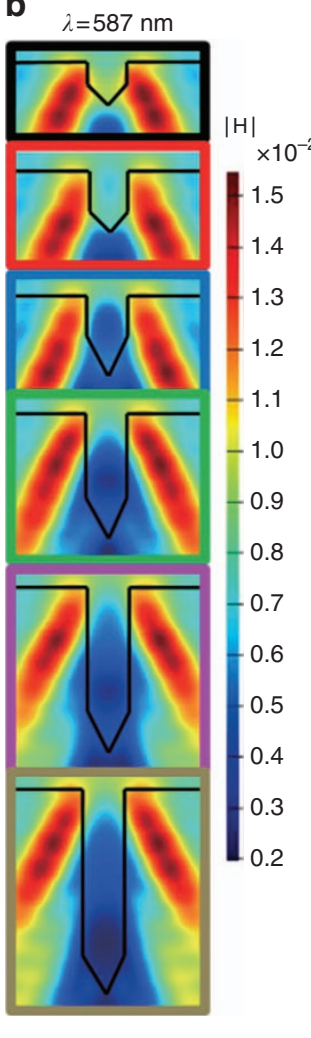

c

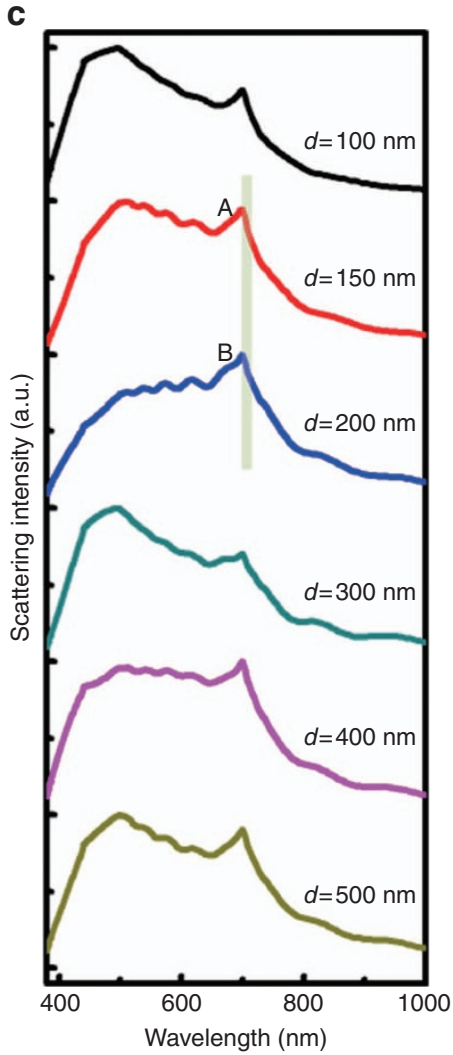

Figure 2 Depth dependence of silicon NGs. (a) Measured scattering spectra of silicon NGs with different depths. The width is fixed at $100 \mathrm{~nm}$. (b) Longitudinal section magnetic field distributions in the $y-z$ plane at $\lambda=587 \mathrm{~nm}$. (c) Calculated scattering spectra of silicon NGs with depths corresponding to the sizes in the experiments.

were used in simulation (see Supplementary Fig. S6b) because the 'dark-field circle' is difficult to simulate. The scattering behaviors can also be adjusted by increasing the width of the silicon NGs, as shown in Supplementary Fig. S7. The scattering signals are increased when widening the groove from $w=100 \mathrm{~nm}$ to $w=400 \mathrm{~nm}$. This phenomenon arises from a larger volume of the submicron cavity, whose mechanism of light confinement is similar to that of Fabry-Perot resonators ${ }^{39,40}$. Usually, the mode volume of Fabry-Perot cavities is larger than $1.6(\lambda / n)^{3}$. Therefore, wider silicon NGs cannot obtain a small mode volume and support LMRMs to realize strong lightmatter interactions.

Although the LMRM as a type of resonant mode that in dielectric materials cannot generate as high of a near-field enhancement as plasmon resonances can to realize strong dipole coupling ${ }^{2}$, the effective overlap and electromagnetic interaction ${ }^{17,22}$ with excitons are expected to generate 'far-field coupling' or 'the coupling of scattering resonances',27. Therefore, for the silicon NG-exciton hybrid system that is scattering-dominant, the constructive or destructive interference between these optical pathways is expected to analyze the interaction characteristics. For a single silicon nanosphere, the interaction between the electric and magnetic dipole can generate directional scattering ${ }^{41}$. For a dipolar source coupled to a magnetodielectric scatterer, directional emission can also be observed $^{42}$. Therefore, we expected that the interference between the J-aggregates (dipole source) and LMRMs (magnetic modes) in the silicon NG would also lead to directional scattering. These phenomena can be explained by Kerker's theory ${ }^{18}$, in which directional scattering could occur depending on the relative strength between the electric dipole mode and the magnetic dipole mode according to the equation below

$$
\sigma_{\text {scat }} \propto \frac{\left(a_{\mathrm{ED}}+b_{\mathrm{MD}} \cos \theta\right)^{2}}{k^{2}}
$$

where $\theta$ is the scattering angle. For forward scattering, $\theta$ equals $0^{\circ}$, and for backward scattering, $\theta$ equals $180^{\circ}$. $a_{\mathrm{ED}}$ and $b_{\mathrm{ED}}$ represent the scattering coefficients contributed by the electric dipole and magnetic dipole. If the dipole moments of the electric mode and magnetic mode are comparable (Figure 3a), then pronounced scattering of the dark state can be produced backward, whereas a strong scattering peak can be seen forward (Figure 3b). If the magnetic mode becomes weaker (see Figure 3c), then the unidirectional scattering effect is weakened (Figure 3d).

To fully understand the interference between J-aggregates and silicon NGs in different directions by simulation, we use bi-pass grooves with a thickness of $100 \mathrm{~nm}$ to replace the actual NGs. The radiation pattern in Figure 4a describes the forward and backward scattering from a gold NG. The localized surface plasmon resonance (LSPR) distributed on the edges interacting with the incident light leads to strong scattering in both the forward and backward directions. Using the Lorentz model to describe the dielectric permittivity of J-aggregates in a simulation, we can investigate the radiation property from this plasmon-exciton hybrid structure as shown in Figure $4 \mathrm{~b}$. At $\lambda=587 \mathrm{~nm}$, J-aggregates with high oscillator strengths filled in the groove experience strong interactions with LSPRs and cause scattering 
a

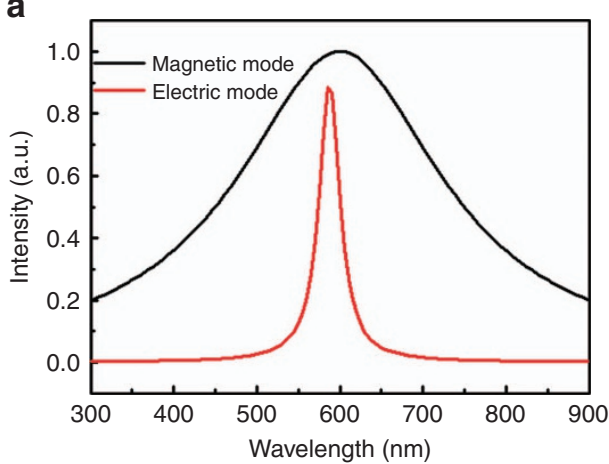

C

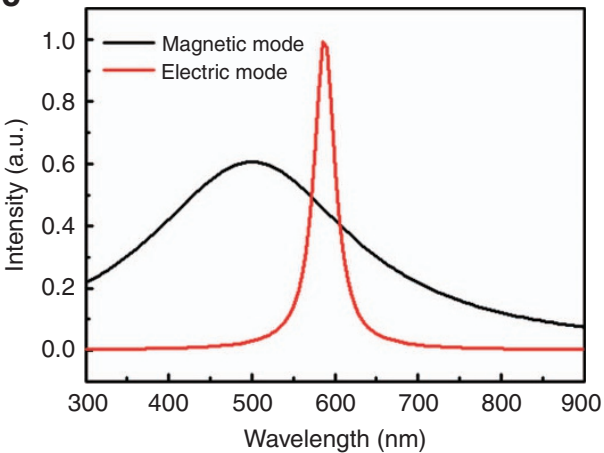

b

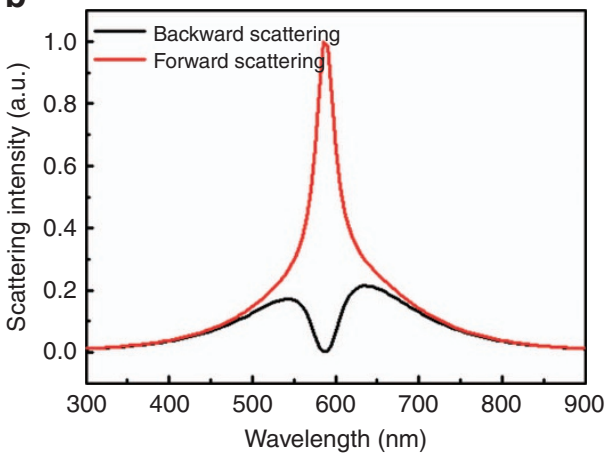

d

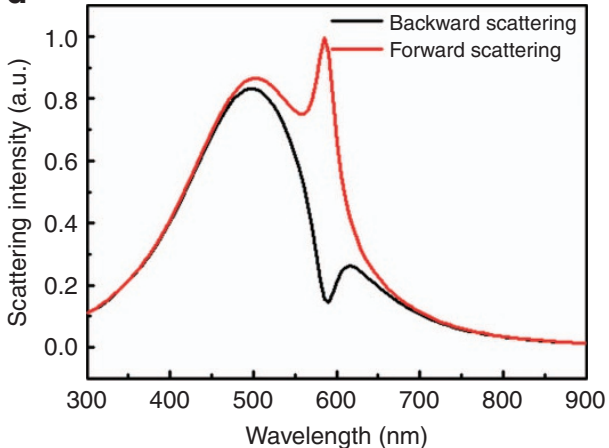

Figure 3 Analysis of unidirectional scattering using the Kerker's theory. (a) and (c) Scattering intensities contributed by electric and magnetic modes with different strengths. (b) Calculated scattering spectra in both forward and backward directions arising from modes in a. (d) Calculated scattering spectra in both forward and backward directions arising from modes in $\mathbf{c}$.

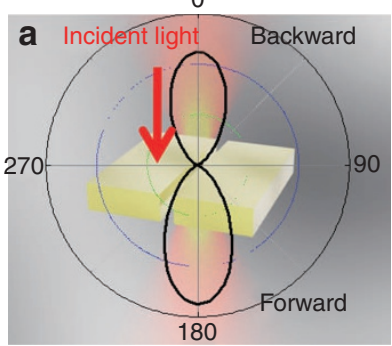

e

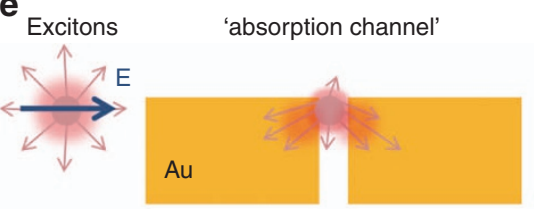

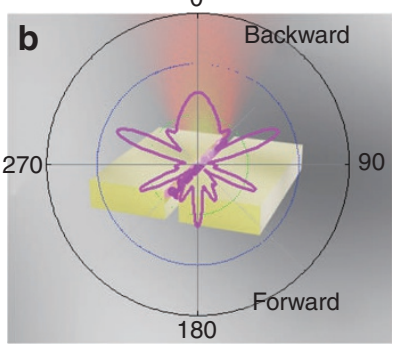

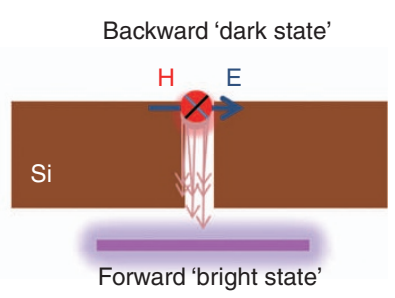

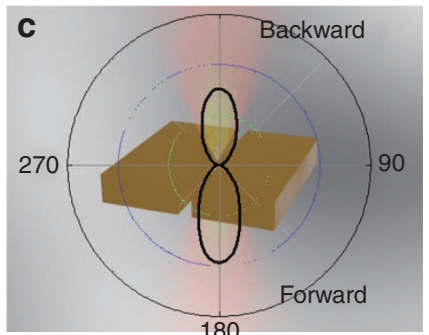

f

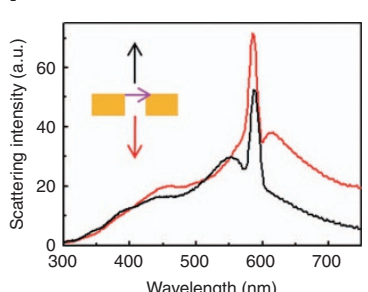

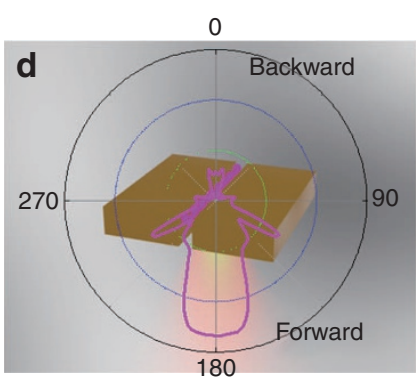

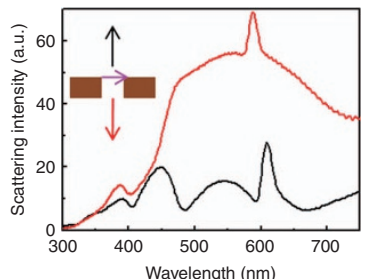

Figure 4 Unidirectional 'dark states' in silicon NGs. (a) The schematic and calculated scattering pattern from a gold bi-pass NG with a thickness of 100 nm under the incident wavelength of $\lambda=587 \mathrm{~nm}$. (b) Schematic illustration and calculated scattering pattern of the Au NG-J-aggregate hybrid nanostructure. (c) Schematic illustration and calculated scattering pattern of a bi-pass Si NG under the incident wavelength of $587 \mathrm{~nm}$. (d) Schematic illustration and calculated scattering pattern of the Si NG-J-aggregate hybrid nanostructure. (e) Schematic explains the 'absorption channel' in a gold-exciton hybrid system and the unidirectional 'dark state' in a silicon-exciton hybrid system. (f) Simulated backward and forward scattering from single-dipole emitter-NG hybrid systems.

quenching. The coupling between J-aggregates and LSPRs mainly occurs on the top surface because the LSPR distributes mainly on the top-surface edges. This interaction directs most uncoupled energies radiating backward, and little light escapes in the forward direction.
The radiation pattern in Figure $4 \mathrm{c}$ exhibits scattering in the forward and backward directions from a bi-pass silicon NG with a thickness of $100 \mathrm{~nm}$. LMRMs interact with the incident light and generate both forward and backward scattering light. Upon filling the groove with 
the J-aggregates, the J-aggregates can be considered electric dipole oscillators interacting with the LMRMs. This situation is exactly Kerker's type of scattering, in which unidirectional scattering can be achieved. As shown in Figure 4d, the scattering can be engineered only toward the forward direction. In Figure 4e, the different interaction mechanisms in gold NGs and silicon NGs is illustrated clearly. The plasmon-exciton coupling process is dominated by an 'absorption channel' because of the strong localized plasmon resonances ${ }^{2,43}$. The energy exchange rate between the excitons and the plasmonic cavity is much higher than are their individual radiative decay rates. However, the situation is different in silicon NGs. The interaction between the excitons and the silicon cavity is realized from the far-field interference between two radiating resonances. On the basis of the unidirectional scattering induced by the electromagnetic interaction, the LMRM cuts off the radiation decay of the J-aggregates and produces a scattering dark state in the backward direction. There is thus no scattering signal found at $\lambda=587 \mathrm{~nm}$ in the backward direction (see Figure $4 \mathrm{~d}$ ). At the same time, strong forward scattering can be obtained through the bipass silicon NG. The silicon NGs in the experiment are single pass, which prevents the leak of uncoupled light forward and forces the uncoupled light to penetrate much deeper into the hybrid structure, which in turn further enhances the coupling between the J-aggregates and silicon NGs. A simulation was also performed by placing a single electric dipole source in the middle of the NGs and calculating the forward and backward scattering spectra as shown in Figure $4 \mathrm{f}$. For the dipole in a gold NG, the forward and backward scattering intensities are nearly equal. For the dipole in a silicon NG, we can see obvious intensity differences between the forward and backward directions, especially at $\lambda=587 \mathrm{~nm}$. In addition, we performed a simulation to explore whether the unidirectional 'dark state' also exists in other silicon nanostructures, such as the silicon nanosphere shown in Supplementary Fig. S8. A silicon nanosphere with a diameter of $140 \mathrm{~nm}$ was chosen whose strong magnetic dipole response is located at approximately $\lambda=587 \mathrm{~nm}$. In addition, the radiation pattern in Supplementary Fig. S8 indicates that no backward scattering suppression occurs because the magnetic modes only distribute inside the sphere and cannot form effective LMRMs for an effective interaction.

In the experiment, after coating a thin-layer TDBC J-aggregate, the scattering spectra of the silicon NGs with different depths were re-measured as shown in Figure 5a. From the AFM characterization in Supplementary Fig. S9, we can see that the thickness of the J-aggregates layer is $\sim 10 \mathrm{~nm}$ and that the J-aggregates distribute on the top of silicon NGs. For a silicon NG with a depth of $100 \mathrm{~nm}$, the scattering spectrum is dominated by a surface-scattering peak below $\lambda=500 \mathrm{~nm}$, which cannot effectively realize overlap with the exciton energy; therefore, only a shallow Fano dip can be observed at $\lambda=587 \mathrm{~nm}$. Meanwhile, for a silicon NG with a depth of $150 \mathrm{~nm}$ whose LMRM can fully overlap with the exciton mode, the significantly suppressed scattering dip at approximately $\lambda=587 \mathrm{~nm}$ reveals an effective destructive interference between the excitons and the silicon NG. When increasing the depth to $200 \mathrm{~nm}$, the red-shifted LMRM weakens the Fano destructive interference between the excitons and the silicon NG; therefore, only a shallow Fano dip can be observed in Figure 5a (blue curve). For a silicon NG with a depth of $300 \mathrm{~nm}$, although the overlap between the resonant energies of the exciton and LMRM can be satisfied in spectrum, the lower overlapping degree in space leads to an incomplete unidirectional 'dark mode' (see radiation patterns in Supplementary Fig. S10). The effect of peak splitting thus becomes slightly weaker (green curve) compared with the NG with $d=150 \mathrm{~nm}$ (red curve). For silicon NGs with depths greater than $400 \mathrm{~nm}$, the nonoverlapping resonant peaks further
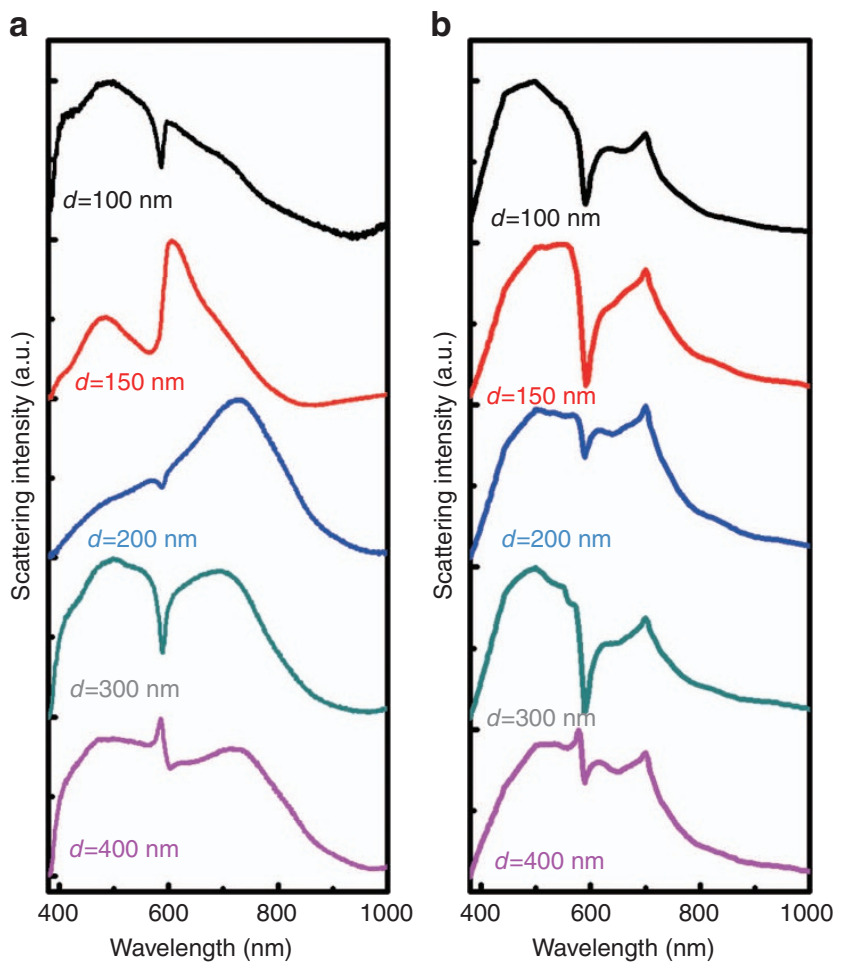

Figure 5 Scattering spectra of J-aggregate-coated silicon NGs. (a) Measured scattering spectra of TDBC J-aggregate-coated silicon NGs with different depths (up to down: $d=100,150,200,300$ and $400 \mathrm{~nm}$ ). (b) Simulated scattering spectra of J-aggregate-coated silicon NGs.

weaken the destructive interference; therefore, only the Fano asymmetric line shape can be observed (pink curve). Using the thickness and location of the J-aggregates obtained from AFM, we simulated the scattering spectra in Figure 5b, which confirm our experimental spectra above. However, some differences between the experiment and simulation still exist that may arise from the optical and geometrical parameters of the J-aggregates used in the simulation not fitting the actual situation accurately.

The scattering spectra of pure J-aggregates and a silicon nanosphere coated with J-aggregates were also measured. For pure J-aggregates, the large harmonic force from the incident field exerts on the J-aggregates directly and leads to the strong backward scattering from the dipole-like excitonic resonance as the measured scattering spectrum shown in Figure 6a. A silicon nanosphere with a diameter of $170 \mathrm{~nm}$ exhibits a broad magnetic dipole resonance in the visible region ${ }^{44}$, and the experimental and simulated scattering spectra are shown in Supplementary Fig. S11. After coating the silicon nanosphere with J-aggregates, we can see a strong and narrow exciton-scattering peak overlapped on the scattering from the nanostructure in the experiment as shown in Figure 6b. We also measured the backward scattering of other sizes of silicon nanospheres spin-coated with J-aggregates. In addition to Figure 6b, the measured scattering spectra in Supplementary Fig. S12 embody strong scattering resonances from excitons. The interaction strength is therefore much lower than that in a J-aggregate-silicon NG system, and the main reason is the different magnetic mode distributions.

The coupled-oscillator model was widely used to analyze the Fano resonances in a system that consists of two interacting oscillators ${ }^{28,29}$. Using the coupled-oscillator model ${ }^{30}$, we can fit the scattering spectra 
a

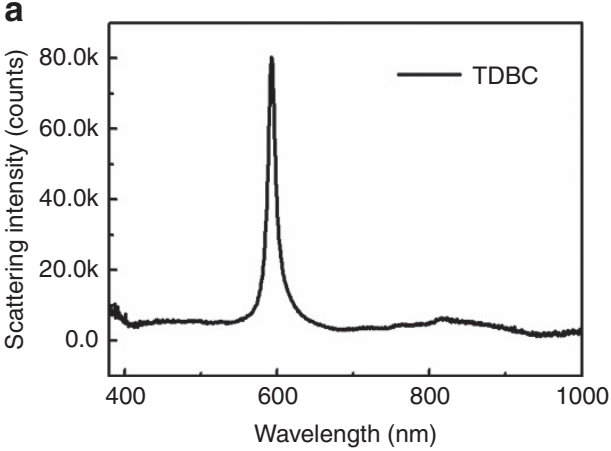

b

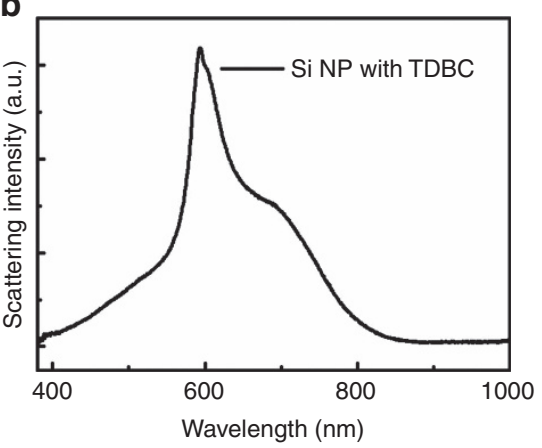

c

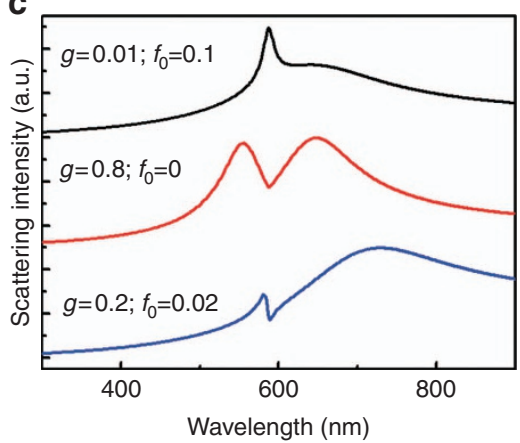

Figure 6 Theoretical analysis of the interaction between silicon nanostructures and excitons. (a) Measured scattering spectrum of pure TDBC J-aggregates layer. (b) Measured scattering spectrum of a J-aggregate-coated silicon nanosphere. (c) The calculated scattering spectra from two interacting oscillators (J-aggregate and Si nanostructures) under different harmonic forces on excitons and different interaction strengths.

of different silicon-exciton systems using different parameters $\left(f_{0}\right.$ and $g$ ) and clarify the general criteria for observing pronounced Fano interference in silicon NGs and silicon nanospheres. The scattering spectra can be described as

$$
\begin{aligned}
& C_{\mathrm{sca}}(\omega)=\frac{8 \pi}{3} k^{4}|\alpha|^{2} \propto \\
& \left|\frac{f_{0} g+f_{\mathrm{NG}}\left(\omega_{0}^{2}-\omega^{2}-i \gamma_{0} \omega\right)}{\left(\omega_{\mathrm{NG}}^{2}-\omega^{2}-i \gamma_{\mathrm{NG}} \omega\right)\left(\omega_{0}^{2}-\omega^{2}-i \gamma_{0} \omega\right)-g^{2}}\right|^{2}+\left|\frac{f_{\mathrm{NG}} g+f_{0}\left(\omega_{\mathrm{NG}}^{2}-\omega^{2}-i \gamma_{\mathrm{NG}} \omega\right)}{\left(\omega_{\mathrm{NG}}^{2}-\omega^{2}-i \gamma_{\mathrm{NG}} \omega\right)\left(\omega_{0}^{2}-\omega^{2}-i \gamma_{0} \omega\right)-g^{2}}\right|^{2}
\end{aligned}
$$

where $\omega_{\mathrm{NG}}$ and $\omega_{0}$ are the resonant frequencies of LMRM and J-aggregate, respectively, and $\gamma_{\mathrm{NG}}$ and $\gamma_{0}$ are the decay rates of LMRM and exciton energies, respectively. $g$ is the interaction strength, and $f_{\mathrm{NG}}$ and $f_{0}$ represent the incident field imposed on the silicon NG and excitons, which describe the scattering excited directly by the incident field. $g$ and $f_{0}$ are two main factors that explain different scattering behaviors in different silicon nanostructures. In our case, the scattering from excitons $\left(f_{0}\right)$ cannot be ignored because considerable J-aggregates have been added in this hybrid system that can generate obvious scattering light around $\lambda=587 \mathrm{~nm}$ under the incident field (see Figure 6a). $f_{0}$ reflects whether excitons couple to the external field or to the NG. If the harmonic force exerting on the excitons approaches zero, then the resonant mode of the excitons can be considered the dark mode. If the harmonic force exerting on excitons is large, then it becomes a bright mode, which hinders the generation of Fano destructive interference.

Interaction strength $g$ determines how pronounced the Fano dip is. It can be given by ${ }^{3,45}$

$$
g \propto N^{\frac{1}{2}} \mu_{\mathrm{J}}\left|E_{\mathrm{vac}}\right| \cdot \frac{k}{f_{0}}=\frac{k \mu_{\mathrm{J}}}{f_{0}} \sqrt{\frac{n_{\mathrm{x}} \hbar \omega}{2 \varepsilon \varepsilon_{0}}} \sqrt{\frac{V_{\mathrm{x}}}{V}}
$$

where $\mu_{\mathrm{J}}$ is the excitonic transition dipole moment, $n_{\mathrm{x}}$ is the density of the J-aggregates, $V_{\mathrm{x}}$ is the result of the overlap integral between the LMRMs and the distribution of J-aggregates and $V$ is the LMRM volume. For dielectric nanocavities, we added coefficient $k / f_{0}$ that represents the contribution of Kerker's scattering from the electromagnetic dipole interaction. It can be seen that the interaction strength is influenced by two aspects. First, the LMRMs interacting with excitons greatly decreased the uncoupled scattering decay backward $\left(f_{0}\right)$ and enhanced the light-matter interaction. Second, such as plasmonic nanocavities, silicon NGs with widths below $100 \mathrm{~nm}$, can realize light trapping under a subwavelength scale. At the same time, unlike solid silicon nanostructures with field enhancements concentrated inside, the leaky magnetic modes diffusing into the groove can fully overlap with the J-aggregates in the groove (large $V_{\mathrm{x}}$ ), which dramatically enhances the ratio $\sqrt{\frac{V_{\mathrm{x}}}{V}}$. These two factors lead to high ' $g$ ' and make silicon NGs realize the Fano-type scattering dark state without strong near-field enhancement.

After knowing the meanings of $g$ and $f_{0}$, we can explain and fit the scattering spectra of different silicon-exciton hybrid systems. Because no effective LMRMs are generated in the silicon nanosphere-exciton hybrid system, the large harmonic force from the incident field exerts on the J-aggregates directly and leads to strong backward scattering from the dipole-like excitonic resonance. In this situation, as shown in the black curve in Figure $6 c, f_{0}$ is large (equals 0.1 ) and $g$ is small (equals 0.1), which exactly describes the situation in the silicon nanosphere coated with J-aggregates (Figure 6b). When an effective electromagnetic interaction occurs between the excitons and the silicon NG, then Kerker's directional scattering can be obtained and uncoupled radiation decay generating backward would be weakened; therefore, the coupling between the incident field and excitons $\left(f_{0}\right)$ becomes much weaker. In contrast, the excitons under unidirectional scattering decay most energies through the silicon nanocavity and boost the interaction strength $(g)$. This interacting case $\left(g=0.8 ; f_{0}=0\right)$ is revealed by the red curve in Figure $6 \mathrm{c}$, which can describe the spectrum from NG with a depth of $150 \mathrm{~nm}$ (Figure 5). When changing the depth of the silicon NG, the Kerker's type unidirectional scattering is weakened; therefore, $f_{0}$ increases to 0.02 and $g$ decreases to 0.2 . We plotted this status in Figure $6 c$ (the blue curve), which is correlated with the scattering spectrum of the silicon NG with a depth of $200 \mathrm{~nm}$. More precise values of the $g$ and $f_{0}$ of different siliconexciton hybrid systems can be deduced from further numerical fitting using the estimated initial values.

Although silicon NGs cannot obtain strong field enhancement, the unidirectional scattering produced by the electromagnetic interaction (small $f_{0}$ ) and the large interaction strength are favorable to obtain a pronounced Fano dip. However, it should be noted that more excitons should be added in this system to realize a strong interaction; therefore, applications such as one-photon nonlinearity ${ }^{46}$ and nonlinear quantum optics ${ }^{39}$ cannot be easily realized. This all-dielectric system also has potential applications in all-optical ultrafast circuits ${ }^{47}$, induced transparency ${ }^{2}$ and the design of novel optical devices with precisely tailored responses ${ }^{48}$.

\section{CONCLUSIONS}

In conclusion, we have investigated the interaction between molecular excitons and resonant modes in silicon NGs. The high-refractive index silicon NG can trap electromagnetic fields on subwavelength scales 
and produce LMRMs. The LMRMs interacting with J-aggregates produce directional scattering and enhance the interaction strength. This new mechanism gives rise to Fano resonances or scattering dark states without large near-field enhancement. By changing the depth of silicon NGs, the scattering spectra of the hybrid system can be engineered. In the emerging applications based on light-matter interactions, silicon NGs provide possibilities to realize on-chip optical processing networks.

\section{ACKNOWLEDGEMENTS}

We thank Hao Wang and Yuchao Li from the School of Physics and Engineering, Sun Yat-sen University for helpful discussions. The National Basic Research Program of China (2014CB931700) and State Key Laboratory of Optoelectronic Materials and Technologies supported this work.

1 Yoshie T, Scherer A, Hendrickson J, Khitrova G, Gibbs HM et al. Vacuum Rabi splitting with a single quantum dot in a photonic crystal nanocavity. Nature 2004; 432: 200-203.

2 DeLacy BG, Miller OD, Hsu CW, Zander Z, Lacey S et al. Coherent plasmon-exciton coupling in silver platelet-J-aggregate nanocomposites. Nano Lett 2015; 15: 2588-2593.

3 Zengin G, Wersäll M, Nilsson S, Antosiewicz TJ, Käll M et al. Realizing strong lightmatter interactions between single-nanoparticle plasmons and molecular excitons at ambient conditions. Phys Rev Lett 2015; 114: 157401.

4 Schlather AE, Large N, Urban AS, Nordlander P, Halas NJ. Near-field mediated plexcitonic coupling and giant Rabi splitting in individual metallic dimers. Nano Lett 2013; 13: 3281-3286

5 Todisco F, D'Agostino S, Esposito M, Fernández-Domínguez Al, De Giorgi M et al. Exciton-plasmon coupling enhancement via metal oxidation. ACS Nano 2015; 9: 9691-9699.

6 Li Q, Wei H, Xu HX. Quantum yield of single surface plasmons generated by a quantum dot coupled with a silver nanowire. Nano Lett 2015; 15: 8181-8187.

7 Manjavacas A, García de Abajo FJ, Nordlander P. Quantum plexcitonics: strongly interacting plasmons and excitons. Nano Lett 2011; 11: 2318-2323.

8 Caldarola M, Albella P, Cortés E, Rahmani M, Roschuk T et al. Non-plasmonic nanoantennas for surface enhanced spectroscopies with ultra-low heat conversion. Nat Commun 2015; 6: 7915 .

9 Kato N, Saito K, Serata T, Aida H, Uesu Y. Morphology and thermochromic phase transition of merocyanine J-aggregate monolayers at the air-water and solid-water interfaces. J Chem Phys 2001; 115: 1473-1484.

10 Yang YM, Wang WY, Boulesbaa A, Kravchenko II, Briggs DP et al. Nonlinear Fanoresonant dielectric metasurfaces. Nano Lett 2015; 15: 7388-7393.

11 Brongersma ML, Cui Y, Fan SH. Light management for photovoltaics using high-index nanostructures. Nat Mater 2014; 13: 451-460.

12 Shi L, Harris JT, Fenollosa R, Rodriguez I, Lu XT et al. Monodisperse silicon nanocavities and photonic crystals with magnetic response in the optical region. Nat Commun 2013; 4: 1904.

13 Shi L, Tuzer TU, Fenollosa R, Meseguer F. A new dielectric metamaterial building block with a strong magnetic response in the Sub-1.5-micrometer region: silicon colloid nanocavities. Adv Mater 2012; 24: 5934-5938.

14 García-Etxarri A, Gómez-Medina R, Froufe-Pérez LS, López C, Chantada L et al. Strong magnetic response of submicron Silicon particles in the infrared. Opt Express 2011; 19: 4815-4826.

15 Albella P, Poyli MA, Schmidt MK, Maier SA, Moreno F et al. Low-loss electric and magnetic field-enhanced spectroscopy with subwavelength silicon dimers. J Phys Chem C 2013; 117: 13573-13584.

16 Shcherbakov MR, Neshev DN, Hopkins B, Shorokhov AS, Staude I et al. Enhanced third-harmonic generation in silicon nanoparticles driven by magnetic response. Nano Lett 2014; 14: 6488-6492.

17 Yan JH, Liu P, Lin ZY, Wang H, Chen HJ et al. Magnetically induced forward scattering at visible wavelengths in silicon nanosphere oligomers. Nat Commun 2015; 6: 7042.

18 Kerker M, Wang DS, Giles CL. Electromagnetic scattering by magnetic spheres. J Opt Soc Am 1983; 73: 765-767.

19 Tian JY, Li Q, Yang YQ, Qiu M. Tailoring unidirectional angular radiation through multipolar interference in a single-element subwavelength all-dielectric stair-like nanoantenna. Nanoscale 2016; 8: 4047-4053.

20 Moitra P, Yang YM, Anderson Z, Kravchenko II, Briggs DP et al. Realization of an alldielectric zero-index optical metamaterial. Nat Photonics 2013; 7: 791-795.
21 Zhao Q, Xiao ZQ, Zhang FL, Ma JM, Qiao M et al. Tailorable zero-phase delay of subwavelength particles toward miniaturized wave manipulation devices. Adv Mater 2015; 27: 6187-6194.

22 Yan JH, Liu P, Lin ZY, Wang H, Chen HJ et al. Directional Fano resonance in a silicon nanosphere dimer. ACS Nano 2015; 9: 2968-2980.

23 Miroshnichenko AE, Kivshar YS. Fano resonances in all-dielectric oligomers. Nano Lett 2012; 12: 6459-6463.

24 Yan JH, Liu P, Lin ZY, Yang GW. New type high-index dielectric nanosensors based on the scattering intensity shift. Nanoscale 2016; 8: 5996-6007.

25 Yang AHJ, Moore SD, Schmidt BS, Klug M, Lipson M et al. Optical manipulation of nanoparticles and biomolecules in sub-wavelength slot waveguides. Nature 2009; 457: 71-75.

26 Ee HS, Kang JH, Brongersma ML, Seo MK. Shape-dependent light scattering properties of subwavelength silicon nanoblocks. Nano Lett 2015; 15: 1759-1765.

27 Hsu CW, Delacy BG, Johnson SG, Joannopoulos JD, Soljacic M. Theoretical criteria for scattering dark states in nanostructured particles. Nano Lett 2014; 14: 2783-2788.

28 Rahmani M, Luk'yanchuk B, Hong MH. Fano resonance in novel plasmonic nanostructures. Laser Photon Rev 2013; 7: 329-349.

29 Joe YS, Satanin AM, Kim CS. Classical analogy of Fano resonances. Phys Scr 2006; 74: 259-266.

$30 \mathrm{Wu}$ XH, Gray SK, Pelton M. Quantum-dot-induced transparency in a nanoscale plasmonic resonator. Opt Express 2010; 18: 23633-23645.

31 Carmeli I, Cohen M, Heifler O, Lilach Y, Zalevsky Z et al. Spatial modulation of light transmission through a single microcavity by coupling of photosynthetic complex excitations to surface plasmons. Nat Commun 2015; 6: 7334

32 Koos C, Vorreau P, Vallaitis T, Dumon P, Bogaerts W et al. All-optical high-speed signal processing with silicon-organic hybrid slot waveguides. Nat Photonics 2009; 3: 216-219.

33 Cai WS, Vasudev AP, Brongersma ML. Electrically controlled nonlinear generation of light with plasmonics. Science 2011; 333: 1720-1723.

34 van de Groep J, Polman A. Designing dielectric resonators on substrates: combining magnetic and electric resonances. Opt Express 2013; 21: 26285-26302.

35 Evlyukhin AB, Reinhardt C, Chichkov BN. Multipole light scattering by nonspherical nanoparticles in the discrete dipole approximation. Phys Rev B 2011; 84: 235429.

36 Li W, Guler U, Kinsey N, Naik GV, Boltasseva A et al. Refractory plasmonics with titanium nitride: broadband metamaterial absorber. Adv Mater 2014; 26 : 7959-7965.

37 Lin HY, Kuo Y, Liao CY, Yang CC, Kiang YW. Surface plasmon effects in the absorption enhancements of amorphous silicon solar cells with periodical metal nanowall and nanopillar structures. Opt Express 2012; 20: A104-A118.

38 Palik ED. Handbook of Optical Constants of Solids, Volume 3. Academic Press: San Diego; 1998.

39 Khitrova G, Gibbs HM, Kira M, Koch SW, Scherer A. Vacuum Rabi splitting in semiconductors. Nat Phys 2006; 2: 81-90.

40 Vahala KJ. Optical microcavities. Nature 2003; 424: 839-846.

41 Fu YH, Kuznetsov AI, Miroshnichenko AE, Yu YF, Luk'yanchuk B. Directional visible light scattering by silicon nanoparticles. Nat Commun 2013; 4: 1527.

42 Rolly B, Geffrin JM, Abdeddaim R, Stout B, Bonod N. Controllable emission of a dipolar source coupled with a magneto-dielectric resonant subwavelength scatterer. Sci Rep 2013; 3: 3063.

43 Blake A, Sukharev M. Surface plasmon polaritons in periodic arrays of V-shaped grooves strongly coupled to quantum emitters. Phys Rev B 2015; 92: 035433.

44 Kuznetsov Al, Miroshnichenko AE, Fu YH, Zhang J, Luk'yanchuk B. Magnetic light. Sci Rep 2012; 2: 492.

45 Eizner E, Avayu O, Ditcovski R, Ellenbogen T. Aluminum nanoantenna complexes for strong coupling between excitons and localized surface plasmons. Nano Lett 2015; 15: 6215-6221.

46 Nan F, Zhang YF, Li XG, Zhang XT, Li H et al. Unusual and tunable one-photon nonlinearity in gold-dye plexcitonic Fano systems. Nano Lett 2015; 15: 2705-2710.

47 Vasa P, Wang W, Pomraenke R, Lammers M, Maiuri M et al. Real-time observation of ultrafast Rabi oscillations between excitons and plasmons in metal nanostructures with J-aggregates. Nat Photonics 2013; 7: 128-132.

48 Lee B, Park J, Han GH, Ee HS, Naylor $\mathrm{CH}$ et al. Fano resonance and spectrally modified photoluminescence enhancement in monolayer $\mathrm{MoS}_{2}$ integrated with plasmonic nanoantenna array. Nano Lett 2015; 15: 3646-3653.

cc)(1) This work is licensed under a Creative Commons AttributionNonCommercial-ShareAlike 4.0 International License. The images or other third party material in this article are included in the article's Creative Commons license, unless indicated otherwise in the credit line; if the material is not included under the Creative Commons license, users will need to obtain permission from the license holder to reproduce the material. To view a copy of this license, visit http:// creativecommons.org/licenses/by-nc-sa/4.0/

(C) The Author(s) 2017

Supplementary Information for this article can be found on the Light: Science \& Applications' website (http://www.nature.com/lsa). 